\title{
Detection and Quantification of Screw-Home Movement Using Nine-Axis Inertial Sensors
}

\author{
Jeong Woo Jeon, Dong Yeop Lee, Jae Ho Yu, Jin Seop Kim, Jiheon Hong \\ Department of Physical Therapy, College of Health Science, Sun Moon University, Asan, Korea
}

Purpose: Although previous studies on the screw-home movement (SHM) for autopsy specimen and walking of living persons conducted, the possibility of acquiring SHM based on inertial measurement units received little attention. This study aimed to investigate the possibility of measuring SHM for the non-weighted bearing using a micro-electro-mechanical system-based wearable motion capture system (MEMSS).

Methods: MEMSS and camera-based motion analysis systems were used to obtain kinematic data of the knee joint. The knee joint moved from the flexion position to a fully extended position and then back to the start point. The coefficient of multiple correlation and the difference in the range of motion were used to assess the waveform similarity in the movement measured by two measurement systems.

Results: The waveform similarity in the sagittal plane was excellent and the in the transverse plane was good. Significant differences were found in the sagittal plane between the two systems $(p<0.05)$. However, there was no significant difference in the transverse plane between the two systems ( $p>0.05)$.

Conclusion: The SHM during the passive motion without muscle contraction in the non-weighted bearing appeared in the entire range. We thought that the MEMSS could be easily applied to the acquisition of biomechanical data on the knee related to physical therapy.

Keywords: Screw-home movement, Biomechanics, Inertial sensor, Micro-Electro-Mechanical System

\section{INTRODUCTION}

Movements of the knee joint were required for various occupational and recreational tasks. ${ }^{1}$ Stability of the knee joint was provided by the bone, articular cartilage, menisci, ligaments, and muscles/tendon units. Disturbance to kinetics and kinematics due to damage or alteration of these structures initiated or accelerated joint instability and degeneration. For this reason, osteoarthritis mainly occurred in the knee synovial joint that could decrease the quality of life. ${ }^{2}$ The movement of sagittal plane accompanied by longitudinal rotation, a phenomenon was "screw-home movement" (SHM) of the knee.,4 This kinematic phenomenon has been known to be the most important factor in providing stability for the knee joint since the 1950s. ${ }^{5}$ Although the biomechanical perspective of SHM established, their values reported varying widely. ${ }^{3,46-11}$ The reason for the

Received Sep 4, 2019 Revised Oct 10, 2019

Accepted Oct 10, 2019

Corresponding author Jiheon Hong

E-mail hgh1020@hanmail.net difference in values of SHM was affected by the various experimental environment, conditions, and measuring equipment..$^{10}$

Since the study of SHM using autopsy specimen, various kinematic studies have been conducted on living persons. ${ }^{6,912}$ Three-dimensional magnetic resonance imaging is a non-invasive method of obtaining precise anatomic and geometric information for in vivo studies of musculoskeletal biomechanics. ${ }^{9}$ However, this method is difficult to reflect dynamic knee movements as a method to analyze a series of images taken under static conditions. ${ }^{6}$ Although the roentgen stereophotogrammetric analysis applied to determine dynamic studies of in vivo knee kinematics, it could lead to unnecessary exposure to radiation. Wearable inertial sensors and the infrared camera were mostly used for 3D motion capture of SHM., ${ }^{4,6}$ The data of the infrared camera was acquired by a triangulation method to calculate the exact position of the marker from images

Copylight $(02019$ The Korean Society of Physical Therapy

This is an Open Access article distribute under the terms of the Creative Commons Attribution Non-commercial License (Http:// creativecommons.org/license/by-nc/4.o.) which permits unrestricted non-commercial use, distribution, and reproduction in any medium, provided the original work is properly cited. 
taken from the surrounding camera. Therefore, it can only be used in a structured environment. ${ }^{12}$ In recent years, microcontroller and nine-axis inertial sensors (miniaturized micro-electro-mechanical systems [EMS] accelerometer, gyroscope, and magnetometer) were introduced as an alternative for human motion capture. ${ }^{12}$ In a previous study, validities of MEMS-based wearable motion capture system (MEMSS) and camera-based motion analysis system were examined. ${ }^{13}$ They reported that there were similarities in flexion/extension waveforms with a high coefficient of multiple correlation (CMC) for whole-body kinematic measurement during walking and stair ascent and descent. However, CMC values for joint angle waveforms associated with rotation and abduction/adduction were much lower.

The acquisition of kinematic data for functional movements was insufficient to confirm the reliability of SHM using MEMSS because of body-weight bearing and effect of muscle contraction. ${ }^{14}$ It is necessary to measure the SHM while minimizing bias by removing muscle contraction and joint load. The objective of the present study was to investigate the possibility of measuring SHM during passive knee movement with non-weight-bearing using a MEMSS.

\section{METHODS}

\section{Participants}

Twenty healthy subjects (twenty males: age, $23.85 \pm 2.08$ years, weight, $73.25 \pm 4.13 \mathrm{~kg}$, height, $173.12 \pm 4.36 \mathrm{~cm}$ ) participated in this study. These subjects had no history of knee joint surgery, neurological disorders, or injuries. Subjects with knee pain, edema, or deformities such as valgus and varus were excluded from this study. The dominant leg was defined as the preferred side when kicking the ball. All the subjects in this study were right leg dominant. This study was conducted after the protocol was approved by the Institutional Review Board of our University.

\section{Experimental data collection}

In this study, MEMSS and camera-based motion analysis systems were used to obtain kinematic data by measuring the movement of the knee joint in sagittal and transverse planes.

\section{1) MEMSS}

Wireless MEMSS (EBIMU24GV3, E2BOX, Seoul, Korea) was used for measurement at a frequency of $40 \mathrm{~Hz}$. To construct segments of thigh and shank, sensors were placed on the lateral side of the thigh (half of the distance between the greater trochanter and lateral epicondyle of the femur) and the proximal medial surface of the tibia shaft, respectively. This placement was selected to minimize skin motion that could affect data because there was not much movement of the muscle. Before performing the passive movement of the knee joint, the subject sat in a chair without a backrest and maintained a full extension position of the knee joint. In this process, the angle of the knee joint in sagittal and transverse planes was defined as zero in the SMULeg software (SMULeg, XENART, Daejeon, Korea). Positive angles indicate flexion and internal rotation, respectively. The MEMSS consists of a triaxial gyroscope $( \pm 2,000 \% \mathrm{sec})$, triaxial accelerometer $( \pm 8 \mathrm{~g})$, and triaxial geomagnetic $( \pm 16 \mathrm{G})$ sensors. The three-dimension orientation was calculated by timeintegrating the gyro output from the conditions provided by the accelerometer and magnetic sensor. The accelerometer provides driftfree inclination. The magnetic sensors are helpful in providing driftfree heading estimates. ${ }^{15}$ The Kalman filter was used to mitigate gyro integration errors by the aiding sensors (accelerometer and magnetic), and to mitigate the errors introduced by the aiding sensors using the gyro output. ${ }^{12}$ The kinematic information of the knee joint obtained from the MEMSS was transmitted to SMULeg software using a receiver. In this software, the angle of the knee joint was analyzed by calculating the difference between the data values of the two sensors attached to the thigh and the shank.

\section{2) Camera-based motion analysis}

In the second method (an optical measurement method), kinematic data during passive movement of the knee joint was obtained using six infrared cameras (Oqus100, QUALISYS, Gothenburg, Sweden). The Qualisys Oqus systems feature high-speed digital cameras with a rate of 250 frames per second (fps) at full resolution and 1,000 fps at reduced resolution. Reflective markers were attached using the Helen-Hayes method. ${ }^{6}$ Position data of reflective markers were obtained at a frequency of $100 \mathrm{~Hz}$. The following two software programs were used for data processing. Qualisys Track Manager (Qualisys, Gothenburg, Sweden) was used to track and collect kinematic data while Visual3D (C-Motion, Rockville, MD, USA) program was used to calculate data. Data were filtered using a secondorder low-pass Butterworth filter with a cut-off frequency of $6 \mathrm{~Hz} .^{16}$ 
Static data were captured while standing in capture volume to define each segment. At this time, the angle of the knee joint in sagittal and transverse planes was defined as zero. Dynamic data were measured during passive movement of the subject's knee joint.

\section{3) Experimental procedure}

For the measurement, the knee was passively moved to the flexionextension by the therapist while the subject was sitting on the chair. To provide stability when the knee joint passively moved, the thigh was fixed with a strap and a metronome was used to keep the velocity of joint movement constant. Joints moved for 2 seconds from the starting position to fully extended position and then back to the starting position for 2 seconds (flexion-extension-flexion passive movement).

\section{Data processing}

During the movement of the knee joint, waveforms similarity of sagittal and transverse plane kinematics were obtained. In order to confirm the similarity of the measured waveforms in the two systems, the knee angle data was normalized to $0-100 \%$ (flexion-extension-flexion). These two normalized waveforms were compared using a $\mathrm{CMC}$ according to previous studies(Figure 1$)^{13}$.

$$
\mathrm{CMC}=\sqrt{1-\frac{\sum_{k=1}^{K}\left[\sum_{p=1}^{P} \sum_{f=\lambda}^{F}\left(\theta_{k p}(f)-\bar{\theta}_{k f}\right)^{2} / K F_{k}(P-1)\right]}{\sum_{k=1}^{K}\left[\sum_{p=1}^{P} \sum_{f=\lambda}^{F}\left(\theta_{k p}(f)-\bar{\theta}_{k}\right)^{2} / K\left(P F_{k}-1\right)\right]}}
$$

$\theta_{k p f}$ is the angle of the knee joint in the frame $f$ measured by the method $p$ (MEMSS or optical motion capture system) at the knee joint motion $k . \bar{\theta}_{k f}$ is the mean angle measured by both systems at frame $f$ in the knee joint motion $k$.

$$
\bar{\theta}_{k f}=\frac{1}{p} \sum_{p=1}^{2} \theta_{k p f}
$$

$\bar{\theta}_{k}$ is the grand mean for the knee joint motion $k$ of the two methods.

$$
\bar{\theta}_{k}=\frac{1}{2 F} \sum_{p=1}^{2} \sum_{f=1}^{F} Y_{k p f}
$$

$P$ is the number of methods $(\mathrm{P}=2)$ and $F$ is the total number of frames $(\mathrm{F}=101) . G$ is the number of knee joint motions.

The range of motion (ROM) in the transverse and sagittal planes during the knee flexion-extension-flexion movement was calculated by subtracting the angle in the flexed position (starting position) from the angle at the fully extended position.

\section{Statistical analysis}

All statistical analyses were performed using SPSS version 22.0 (IBM Co., Armonk, NY, USA). Independent t-test was performed to compare differences in ROM of both systems. The statistical signifi-
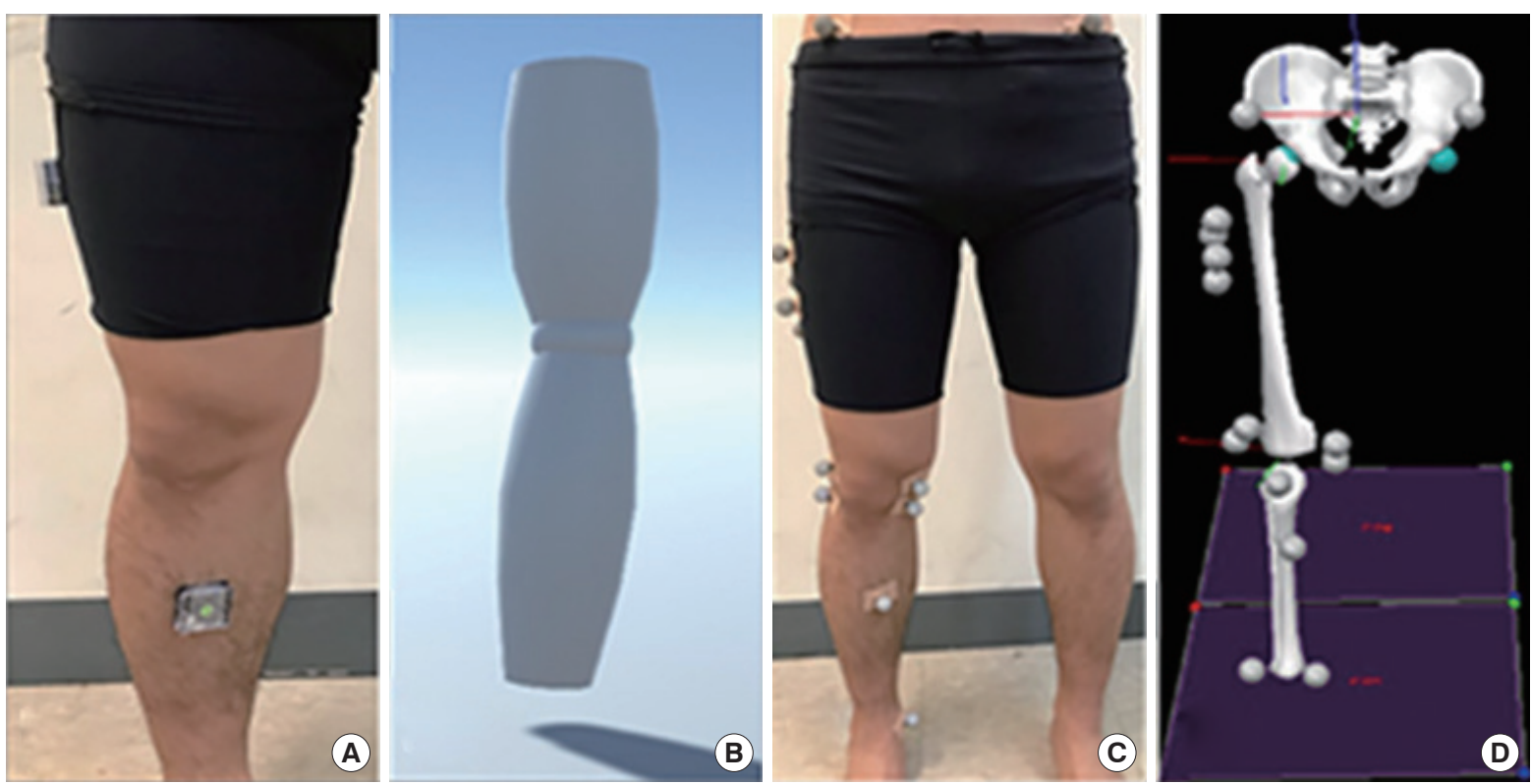

Figure 1. (A) Placement position of the MEMSS sensor. (B) Modeling in the SMULeg software. (C) Placement of the reflective marker in the optical measurement. (D) Modeling in the Visual $3 \mathrm{~d}$ software. 
cance level was set at $\mathrm{p}<0.05$. Waveform similarity was assessed using a CMC. CMC values were interpreted as shown below: weak, $<0.65$, moderate, 0.65-0.75, good, 0.75-0.85, very good, 0.85-0.95, excellent, $>0.95 .^{17}$

\section{RESULTS}

Figure 2 shows the waveform similarity of the knee joint movement in the sagittal plane measured by both systems during the starting position to the full extension position and then back to the starting position. The waveform similarity was excellent $(\mathrm{CMC}=0.959)$. The maximum extension of the knee joint was found in $49 \%$ and $51 \%$ of normalized values in the optical motion capture system and MEMS, respectively. Figure 3 shows the waveform similarity of the knee joint movement in the transverse plane measured by both systems. The waveform similarity was good $(\mathrm{CMC}=0.803)$. The maximum external rotation of the knee joint was found in 49\% and 56\% of normalized values in the optical motion capture system and

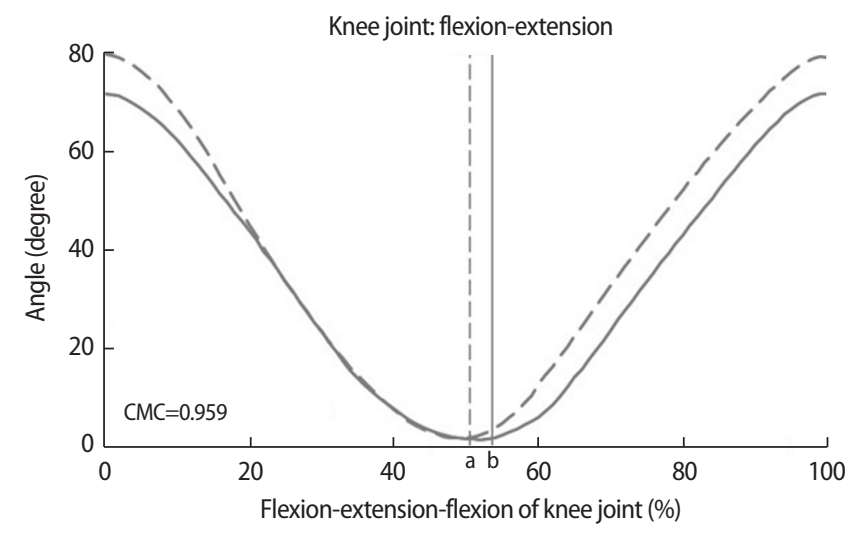

Figure 2. Waveform similarity of the knee joint movement in the sagittal plane measured by both systems. The solid line represents the angle measured by the MEMSS and the dashed line represents the angle measured by the optical motion capture system. The knee joint motion (\%) means the flexion-full extension-flexion movement $\left(0^{\circ}=\right.$ full extension). a: The maximum angle of the knee extension in the optical motion capture system (49\%). b: The maximum angle of the knee extension in the MEMSS (52\%).
MEMS, respectively.

Table 1 shows differences in ROM in sagittal and transverse plane kinematics of the knee joint measured by both systems. Significant differences were found in the sagittal plane kinematics of knee joints between the two systems $(\mathrm{p}<0.05)$. However, there was no significant difference in transverse plane kinematics between the two systems $(p>0.05)$.

\section{DISCUSSION}

In this study, we investigated the possibility and reliability of SHM acquisition using MEMSS in normal subjects. The variation of SHM was obtained at $12.64^{\circ}$ using MEMSS and $14.79^{\circ}$ using an optical motion capture system while the knee was fully extended at about $70^{\circ}$ flexion position. Besides, there was no statistically significant difference in the variation of SHM between MEMSS and the optical motion capture system. Although SHM was an important factor in providing stability to the knee joint, it was difficult to show

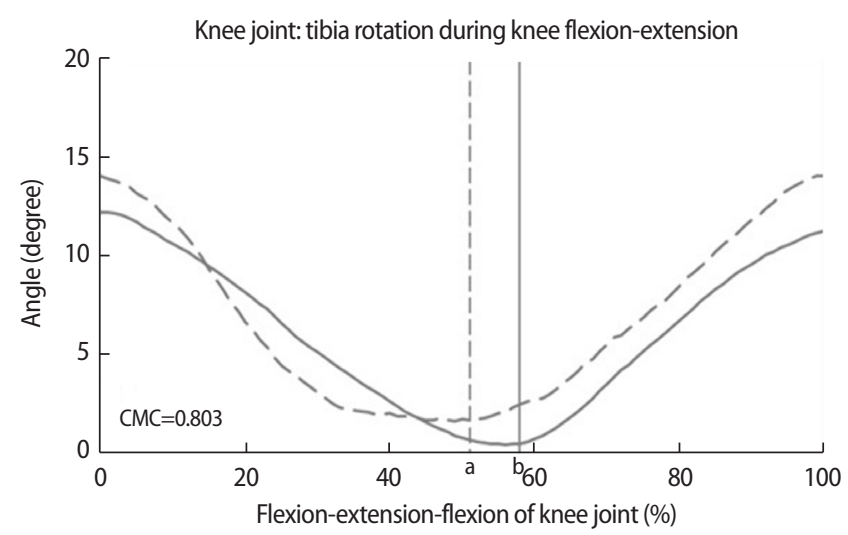

Figure 3. Waveform similarity of the knee joint movement in the transverse plane measured by both systems. The solid line represents the angle measured by MEMSS and the dashed line represents the angle measured by the optical motion capture system. The knee joint motion (\%) means the flexion-full extension-flexion movement $\left(0^{\circ}=\mathrm{ex}^{-}\right.$ ternal rotation of tibia). a: The maximum angle of the tibia external rotation in the optical motion capture system (49\%). b: The maximum angle of the tibia external rotation in the MEMSS (56\%).

Table 1. Comparison of difference in ROM of the knee joint measure by optical motion capture system and MEMSS

\begin{tabular}{lcc}
\hline \multirow{2}{*}{ Movement of the knee joint } & \multicolumn{2}{c}{ Measurement System $\left(^{\circ}\right.$ ) } \\
\cline { 2 - 3 } & Optical motion capture system & MEMSS \\
\hline Sagittal plane (Flexion-Extension) & $81.37 \pm 9.77$ & $72.68 \pm 6.15$ \\
Transverse plane (Tibia Rotation) & $14.79 \pm 6.62$ & $12.64 \pm 4.61$
\end{tabular}

All results are expressed as mean \pm standard deviation.

ROM: range of motion, MEMS: micro electro-mechanical system.

${ }^{*} p<0.05$. 
the correct amount of SHM due to various factors. ${ }^{5,10,18}$ In previous studies using various measuring instruments such as testing rig for in vitro measurements, marker-based 3D-camera systems, and 2D$3 \mathrm{D}$ image matching procedure, the range of $\mathrm{SHM}$ varied from $5^{\circ}$ to $36^{\circ}$ during the extension of the knee joint. ${ }^{3,4,6,79-11}$ Hacker et al. ${ }^{18}$ reported that $\mathrm{SHM}$ was less than $30^{\circ}$ as a result of a meta-analysis of 19 publications. In their study, an analysis of 14 in vitro studies showed that most SHM appeared in the range of full extension from the knee joint flexion at $30^{\circ} .^{18}$ In contrast, a relatively small SHM showed in the present study. It occurred during the entire range of knee extension and flexion.

Previous studies reported that the SHM phenomenon was different depending on the presence or absence of the knee joint load. ${ }^{10,19}$ The tibia followed a consistent path relative to the femur when there was an unloaded knee joint. ${ }^{10}$ Consequently, the SHM appeared at the last extension of the loaded knee whereas the SHM appeared continuously over the entire range at the unloaded knee. In the current study, the SHM was shown in the whole range because it was a passive movement in a sitting position without weight on the knee. In a previous study measuring the rotation of the knee joint using an optical motion capture system during functional walking, the external rotation was observed about $10^{\circ}$ during swing phase without weight-bearing. ${ }^{6}$ Interestingly, in this previous study, external rotation occurred at about $5^{\circ}$ when the knee was bent from $0^{\circ}$ to $15^{\circ}$ (loading response phase), a reversal of the screw-home mechanism. ${ }^{6}$ This result suggests that the SHM significantly affected by body weight.

In the current study, results of MEMSS showed excellent reliability in the sagittal plane $(\mathrm{CMC}=0.957)$ and good reliability $(\mathrm{CMC}=$ 0.803 ) in the transverse plane compared to the optical motion capture system. The CMC was useful for confirming the similarity of joint angle waveforms between data acquired by other equipment. ${ }^{13,20}$ In previous studies, three-dimensional kinematic data of lower limbs were compared with Xsens MVN BIOMECH based on inertial sensor and camera-based analysis system. ${ }^{13}$ The previous study showed that $\mathrm{CMC}$ value of the flexion/extension of the knee joint was high in walking while the value of rotation was relatively low. ${ }^{13}$ consistent with results of the present study. Brennan et al. ${ }^{21}$ reported that difference of kinematic data for the same motion trail in two different systems was caused by technologies, definitions of anatomical frames, or simply two identifications of anatomical land- marks. In this study, the same anatomical landmarks were used. However, differently defined axes were used in anatomical frames between the two systems. The main effect of differently defined rotational axes was on the angle, although the overall pattern of change was similar. ${ }^{13,21}$ Therefore, in this study, angular variation was used for SHM to reduce the influence. As a result of obtaining SHM using the two systems, there was no significant difference in total variation or good reliability.

The limitations of this study are as follows. First, the markers for the two measuring devices were completely attached to the skin using double-sided tape, but the influence of soft tissues should be considered. Second, this study did not measure SHM under various conditions. In a further study, it is necessary to accurately identify the factors affecting the value through SHM acquisition in various situations.

\section{CONCLUSION}

We investigated the possibility of obtaining a variation of SHM using MEMSS. We performed passive knee motion in the absence of weight to reduce the error of the experiment. In this study, the variation of SHM was obtained at $12.64^{\circ}$ in the whole range while the knee was fully extended at about $70^{\circ}$ flexion position. The mechanism for generating the SHM in the non-weighted bearing might be to follow a consistent path because of the geometry and shape of the articular surfaces. To the best of our knowledge, this study confirmed the possibility of analyzing biomechanical information related to physical therapy using an inertial sensor developed in Korea.

\section{ACKNOWLEDGEMENTS}

This study was supported by National Research Foundation of Korea Grant funded by the Korean Government (NRF-2016R1D1A3B03932586).

\section{REFERENCES}

1. Moglo KE, Shirazi-Adl A. Cruciate coupling and screw-home mechanism in passive knee joint during extension-flexion. J Biomech. 2005; 38(5):1075-83.

2. Pereira D, Peleteiro B, Araujo J et al. The effect of osteoarthritis definition on prevalence and incidence estimates: a systematic review. Osteoarthri- 
tis Cartilage. 2011;19(11):1270-85.

3. Hallen LG, Lindahl O. The "screw-home" movement in the knee-joint. Acta Orthopaedica Scandinavica. 1966;37(1):97-106.

4. Piazza SJ, Cavanagh PR. Measurement of the screw-home motion of the knee is sensitive to errors in axis alignment. J Biomech. 2000;33(8):102934.

5. De Palma A. Diseases of the Knee. Philadelphia, Lippincott Co. 1954.

6. Kim HY, Kim KJ, Yang DS et al. Screw-home movement of the tibiofemoral joint during normal gait: three-dimensional analysis. Clin Orthop Surg. 2015;7(3):303-9.

7. Matsumoto H, Seedhom BB, Suda Y et al. Axis location of tibial rotation and its change with flexion angle. Clin Orthop Relat Res. 2000;371:17882.

8. Patel VV, Hall K, Ries M et al. A three-dimensional MRI analysis of knee kinematics J Orthop Res. 2004;22(2):283-92.

9. Samukawa M, Yamamoto T, Miyamoto S et al. Analysis of tibial rotation using magnetic resonance imaging. Man Ther. 2009;14(6):712-5.

10. Wilson DR, Feikes JD, Zavatsky AB et al. The components of passive knee movement are coupled to flexion angle. J Biomech. 2000;33(4): 465-73.

11. Zhang LK, Wang XM, Niu YZ et al. Relationship between patellar tracking and the "Screw-home" mechanism of tibiofemoral joint. Orthop Surg. 2016;8(4):490-5.

12. Brigante $\mathrm{CM}$, Abbate N, Basile A et al. Towards miniaturization of a MEMS-based wearable motion capture system. IEEE Transactions on
Industrial Electronics. 2011;58(8):3234-41.

13. Zhang JT, Novak AC, Brouwer B et al. Concurrent validation of Xsens MVN measurement of lower limb joint angular kinematics. Physiol Meas. 2013;34(8):N63-N69.

14. Victor J, Labey L, Wong P, et al. The influence of muscle load on tibiofemoral knee kinematics. J Orthop Res. 2010;28(4):419-28.

15. Sabatini AM. Variable-state-dimension kalman-based filter for orientation determination using inertial and magnetic sensors. Sensor. 2012; 12(7):8491-506.

16. King SL, Barton GJ, Ranganath LR. Interpreting sources of variation in clinical gait analysis: A case study. Gait Posture. 2017;52:1-4.

17. Ferrari A, Cutti AG, Garofalo P et al. First in vivo assessment of "Outwalk": a novel protocol for clinical gait analysis based on inertial and magnetic sensors. Med Biol Eng Comput. 2010;48(1):1-15.

18. Hacker SP, Ignatius A, Durselen L. The influence of the test setup on knee joint kinematics-A meta-analysis of tibial rotation. J Biomech. 2016;49(13):2982-8.

19. Blankevoort L, Huiskes R, de Lange A. The envelope of passive knee joint motion. J Biomech. 1988;21(9):705-20.

20. Roislien J, Skare O, Opheim A et al. Evaluating the properties of the coefficient of multiple correlation (CMC) for kinematic gait data. J Biomech. 2012;45(11):201418.

21. Brennan A, Deluzio K, Li Q. Assessment of anatomical frame variation effect on joint angles: A linear perturbation approach. J Biomech. 2011; 44(16):2838-42. 